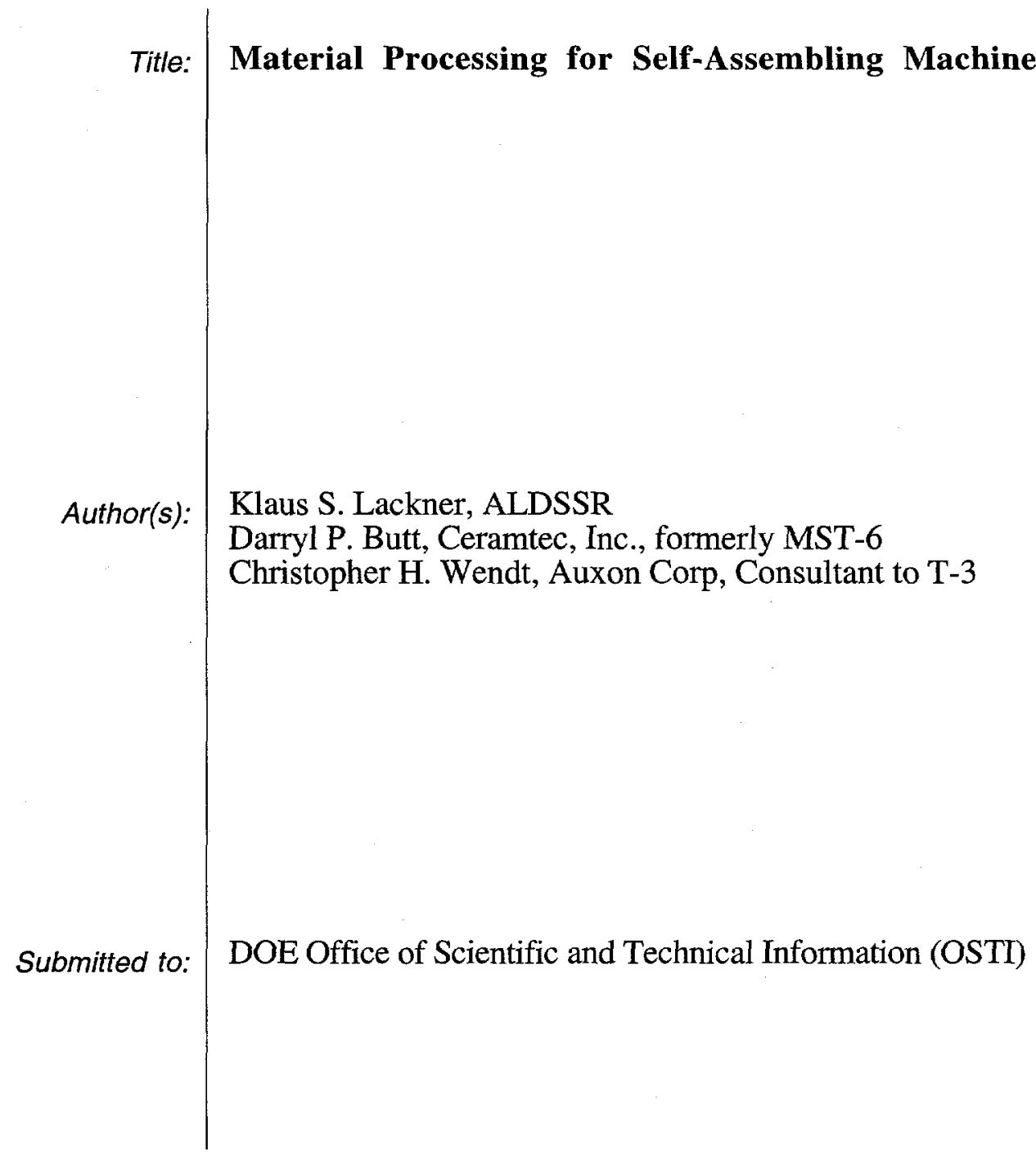

Los Alamos National Laboratory, an affirmative action/equal opportunity employer, is operated by the University of California for the U.S. Department of Energy under contract W-7405-ENG-36. By acceptance of this article, the publisher recognizes that the U.S. Government retains a nonexclusive, royaltyfree license to publish or reproduce the published form of this contribution, or to allow others to do so, for U.S. Government purposes. Los Alamos National Laboratory requests that the publisher identify this article as work performed under the auspices of the U.S. Department of Energy. Los Alamos National Laboratory strongly supports academic freedom and a researcher's right to publish; as an institution, however, the Laboratory does not endorse the viewpoint of a publication or guarantee its technical correctness. 


\section{DISCLAIMER}

This report was prepared as an account of work sponsored by an agency of the United States Government. Neither the United States Government nor any agency thereof, nor any of their employees, make any warranty, express or implied, or assumes any legal liability or responsibility for the accuracy, completeness, or usefulness of any information, apparatus, product, or process disclosed, or represents that its use would not infringe privately owned rights. Reference herein to any specific commercial product, process, or service by trade name, trademark, manufacturer, or otherwise does not necessarily constitute or imply its endorsement, recommendation, or favoring by the United States Government or any agency thereof. The views and opinions of authors expressed herein do not necessarily state or reflect those of the United States Government or any agency thereof. 


\section{DISCLAIMER}

Portions of this document may be illegible in electronic image products. Images are produced from the best available original document. 


\title{
Material Processing for Self-Assembling Machine Systems
}

\author{
Klaus S. Lackner*, ALDSSR \\ Darryl P. Butt, Ceramtec, Inc., formerly MST-6 \\ Christopher H. Wendt, Auxon Corp, Consultant to T-3
}

\begin{abstract}
We are developing an important aspect of a new technology based on selfreproducing machine systems. Such systems could overcome resource limitations and control the deleterious side effects of human activities on the environment. Machine systems capable of building themselves promise an increase in industrial productivity as dramatic as that of the industrial revolution. To operate successfully, such systems must procure necessary raw materials from their surroundings. Therefore, next to automation, most critical for this new technology is the ability to extract important chemicals from readily available soils. In contrast to conventional metallurgical practice, these extraction processes cannot make substantial use of rare elements. We have designed a thermodynamically viable process and experimentally demonstrated most steps that differ from common practice. To this end we had to develop a small, disposable vacuum furnace system. Our work points to a viable extraction process.
\end{abstract}

\section{Background and Research Objectives}

Manufacturing technology and automation have advanced steadily over the course of the last century. Today, the level of automation that can be achieved is astonishingly high. Wherever the cost of human labor is high, automation has reached extraordinary levels. For example, involving people in the production of computer chips is costly because their presence requires substantial investment into clean room technology. Consequently, the process of making computer chips has been nearly fully automated. There are many other example which suggest that most any manufacturing, machining or assembly process used in today's practice could be automated with today's level of technology, even though in many instances the economics would not yet justify the effort.

This raises the question whether one could design a factory that does not require human input. In general, the design of such a plant is considered feasible, even though with today's technology it would again not yet be economical. Once such a plant can be built, the next obvious question would be whether the machines and components required for this plant could also be manufactured in a fully automated plant.

We refer to this concept as recursive automation; it also has been discussed as superautomation. Recursive automation would drastically change the overall economics of

*Principal Investigator, e-mail: ksl@lanl.gov 
the automation concept. In this context the first step of a fully automated manufacturing plant becomes a critical threshold that needs to be overcome. Beyond this threshold the economics of the approach is likely to improve rapidly.

If recursive automation can be demonstrated, one could envision a manufacturing system that functions essentially without human input. Obviously many of the machines involved in such a system would be considered robots and therefore this discussion returns us back to a question which was raised first by the famous mathematician John von Neumann: Can a robot be designed that is capable of building a copy of itself?

Von Neumann, at least in principle, answered this question in the positive [3]. In contrast to the universal computer, he postulated the universal robot, with one of its defining features being the ability of building a copy of itself. At the time, in the early fifties, an actual implementation was not attempted and from today's point of view would probably not have been possible. Nevertheless, the mathematical analysis still stands. Today, with self-replicating computer virus, the concept has gained in credibility. Note, too, that nano-technology, with its self-assembly, presupposes the existence of such a technology. Here, however, we prefer to only look at the possibility of self-replicating machine systems. The miniaturization of nano-technology requires an additional set of innovations we are not at all addressing here.

The technological and social consequences of a machine system that can build itself were immediately appreciated by a number of visionaries. Already in the late fifties E. F. Moore [4], described a large system of small ocean going units that would extract raw materials from ocean water, build more of themselves and eventually deliver raw materials of practical use to the shore. In essence, each unit was a complete self-replicating unit and the systems input was solar energy and minerals dissolved in ocean water.

Freeman Dyson [5] described a self-replicating system and its implications on society in the seventies. Later in the eighties, NASA undertook an extensive study [6, 7] that suggested that self-replicating machine systems could be implemented on the moon or other planets.

All these groups realized that self-replicating, self-sustaining machine systems would break the relation between the amount of output product and the input of human labor. If the human effort in producing a new product is limited to the design phase, after which the system, in an autonomous fashion, can build itself up to any desired size, then the input of human labor becomes independent of the size of the output.

A first preview of how such an industrial society might operate, we can already see in the example of computer software. For software, to a good approximation the input of human labor is set by the software development and the marginal cost of yet another copy 
is practically nil. The effect this new technology has had on the US economy cannot be underestimated, and what we are proposing here is looking for a similar or even greater impact on the technology of producing tangible goods rather than just computer codes.

Self-replicating machine systems could have a major impact on the world economy. They would result in a productivity increase that would dwarf the industrial revolution. As a consequence one could afford to spend a much larger effort on production than is costeffective today, and many of the deleterious side effects of today's industry could simply be eliminated as one could afford to remedy these problems. For this to work, the system would need to be able to build itself, provide itself with virtually unlimited material and energy resources, and then from this position provide raw materials, energy, and manufactured goods for people.

As a basis for our research, we had developed new insights into this technology (1, 2). In contrast to NASA we had focussed our effort on terrestrial applications. Our basic question is whether it is possible with today's technology to develop a self-replicating machine system that can build more or copies of itself using readily available raw materials and energy. While we are aware of the fact that proving the feasibility of such a system requires an actual implementation that solves all the problems of automation, we have shown that the system can be developed in pieces.

We consider the issue of automation as one that can only be addressed by actually designing the system. This would far exceed the scope of the effort we are prepared to mount today. Consequently we have focused on the potential technological bottlenecks that can be identified from the start. A major one is the extraction and processing of raw materials. Today's level of technology rules out intelligent processes searching for valuable ores, instead such a system would be forced to utilize readily available raw materials, i.e. soils or raw dirt. While it is easy to collect such materials, their processing represents a major challenge.

Because it is one of the major stepping stones towards a working system, our work has focused on the material processing aspects. They also played a major role for the NASA system. Yet, our approach differs drastically from the one NASA has taken. Its advantage is that it resolves a major problem that was not addressed in the earlier NASA study. Utilizing extremely rare elements like fluorine in the extraction process will greatly limit the rate at which such a system can grow. Since the extraction process is a large part of the total system, a hydrofluoric acid bath as suggested by NASA will represent a large fraction of the total mass of the growing system. Thus the production of hydrofluoric acid will become a rate limiting process. Our approach in contrast is based on developing chemical processes that completely avoid the need for rare elements. 


\section{Importance to LANL's Science and Technology Base and National R\&D Needs}

The work developed here is basic research that provides insights into the fundamental processes of automation, chemical processing and the design of industrial processes. The original paper that outlines our basic ideas has also laid the corner stone for a growing effort in carbon management at the Laboratory. In this sense, this LDRD has started a new program development effort, which is already bearing fruit.

The concepts of recursive automation and superautomation will play a critical role in the productivity and competitiveness of industrial nations. Economic strength of the nation is one major goal for the Laboratory and this is our motivation to be involved in this project. In some sense, gradual improvements and progress in the automation of industries brings about superautomation, even if it is never stated as an explicit goal. Robots are already assembled by very similar robots. However, such a laissez faire approach amounts to an ad-hoc and piecemeal implementation by accident. As industries are gradually moving to ever-higher levels of automation, superautomation becomes the unavoidable consequence. The nation that first arrives at a system so highly automated that to a good approximation it builds and maintains itself has an enormous competitive advantage. As mentioned above, automation will increase productivity and once a threshold is overcome the increase will become rapid and dramatic. Rather than relying on unfocussed undirected progress, we are developing a focussed effort beyond just automating each step. Our goal is to develop the basic concepts. This is high risk, fundamental research motivated by its usefulness for dramatic economic growth in the future.

\section{Scientific Approach and Accomplishments}

Our goal is to establish recursive automation as a viable approach to increased productivity. Ultimately this will lead to self-maintaining and self-producing machines. Specifically, we are developing the material processing technology required in such a system. In order to motivate our approach we summarize our results on self-replicating machinery which we have published as "Exponential Growth of Large Self-Replicating Machine Systems", Ref. 1.

Automation in the manufacture of industrial and agricultural goods has progressed during this century to a remarkable degree. While at the beginning of the century it took a large portion of the population simply to grow food, today this portion is a few percent. Automated factories are becoming reality. Fully automated production lines for solar cells already exist. Indeed, it is hard to find a manufacturing process that could not be 
automated - at least in principle - using today's technology base. Judging from examples of state of the art in automation, it appears that the individual production steps that are anticipated in a self-replicating system can be automated following industrial practice. In our paper, we have shown that the fundamental constraints on a self-replicating system can be satisfied and that by starting with today's level of technology one can use recursive automation to implement such a system step by step. For each production chain, which starts with the raw inputs and ends in a single product, we have identified necessary and sufficient productivity requirements, which establish the minimum productivity of each step and limit the number of steps in a single production chain. Whether complete automation is feasible has been reduced to the satisfaction of these requirements for each individual chain.

For a self-replicating machine system, the object is to achieve exponential growth so that it can eventually attain a very large size. Ultimately, all significant inputs required for the growth of the system must be procured by the system itself from its natural surroundings. Thus, inputs are restricted to the most easily accessible ones, which for a land-based system are raw dirt, air and sunlight. The minimal system would contain solar cells whose energy output would be fed to a number of automated material gathering, processing and assembly units, which we call auxons. The auxons, in turn, would produce additional solar cells and more auxons. Once the design size is reached, nearly the entire power generating and material processing capacity could be diverted to perform useful tasks.

An auxon system could be broken up into machines (auxons) and a fixed infrastructure, which would include almost complete coverage of an area by solar energy collectors, a grid of tracks for transportation and some form of energy distribution. The infrastructure would provide a controlled environment for the auxons, which would be specialized machines with the minimum level of sophistication necessary to accomplish their assigned tasks. Whenever possible, auxons would use physical constraints and simple rules for their operation. In this sense they would be similar to machines on the factory floor. However, the system would have to be sufficiently open so that its neighbors could easily absorb the effects of a failure of a single auxon.

A large portion of an auxon system's production capability would be dedicated to processing its material input of raw dirt and extracting the elements required for the growth of the system. The typical composition of raw dirt is such that all elements necessary for building an auxon system are available and can be used in roughly the proportions in which they occur. The constraints of a self-reproducing system forced us to design a novel metallurgical process for the element extraction, which is shown in Figure 1. The difference relative to conventional metallurgy is not only the lower concentration of values 
but also the avoidance of certain scarce elements like fluorine, which are commonly used in mineral extraction processes. The goal of this proposal is to optimize the chemical extraction processes and to demonstrate their viability.

Under reasonable assumptions concerning the energy collection efficiency (10\%) and the system mass per unit area $\left(10 \mathrm{~kg} / \mathrm{m}^{2}\right)$, the solar energy available per unit area is sufficient for such a system to double its size in a matter of months. Hence it can grow to a very large size within a few years. An increase in size by a factor of $10^{8}$ within a decade appears to be achievable. At $10^{6} \mathrm{~km}^{2}$, which is comparable in size to half the cropland in the United States or one tenth of the Sahara, the electric energy production from an auxon system would be about twenty-five times today's worldwide energy production.

In outlining a strategy for research in this field, the work that needs to be done can be broken up into immediate, intermediate and long term goals. The immediate goal must be to establish experimentally and support theoretically the feasibility of the auxon concept. Of the fundamental issues that must be addressed, the most critical is that of the separation of raw dirt into useful materials. This is what we have studied under the auspices of this LDRD.

Unlike automation, which is certainly possible in principle and ultimately can only be demonstrated by building a complete system, the chemistry of material separation and processing is a fundamental issue that must be resolved regardless of the details of the implementation. In the intermediate future, subsequent work will have to demonstrate the automation concept. The performance of representative manufacturing chains must be quantified in order to establish the feasibility of a growing system. Finally, a more detailed picture of the complete system must be developed. Through a modest investment, one will establish whether a large commitment to this new technology is justified and is likely to bear fruit.

On an intermediate time scale, future efforts should also focus on applications for recursive automation. Because the input of human labor and capital is reduced as more and more of a factory's equipment is produced internally, it is likely that recursive automation will have commercially viable applications long before a self-replicating machine system can be constructed. Such applications are likely to be worked out in collaboration with industry.

There are several criteria for selecting an application for recursive automation. Clearly any application should be relevant to the design of a complete auxon system and for a first attempt the task performed should not be too complex. Furthermore, the potential demand for a product which is manufactured using recursive automation must be large, in order to justify the automated mass production of the manufacturing equipment. Finally, 
the value added in the processing must be large compared to the input costs, otherwise the potential for cost savings from recursive automation is not very significant. The last constraint is most easily achieved with low-grade, low-cost inputs. This indicates that the type of separation chemistry we study is likely to be relevant to recursively automated processing plants even though they still fall far short from complete automation.

The first applications for recursive automation are likely to be found among known technologies whose implementations would be desirable but which are too expensive to compete with cheaper less desirable alternatives. The collection of solar energy with photovoltaic cells and the liquefaction of coal are two examples. The former would tap a virtually limitless energy source that avoids the use of fossil fuels altogether, while the latter would make a large amount of domestic coal accessible for use in automobiles. Neither process is technologically out of reach, but both are too expensive to compete with cheap foreign oil. Since similar processes will also have to be incorporated into a complete auxon system, such work can be relevant to near term applications without sacrificing progress towards the ultimate goal.

Once energy collection has reached the point that an incomplete auxon system can provide its own energy needs, there are more applications. For example, the desalination of seawater could provide a limitless supply of water for agriculture. The extraction of aluminum, titanium and possibly other values from low-grade ores are applications which would also further the development of a complete auxon system. Auxon systems could process ores that would be considered useless at today's extraction costs. As the state of the art in auxon technology progresses, ore composition would differ less and less from ordinary crustal abundances. Thus one opens another limitless resource.

The ultimate goal of the auxon project is to create self-replicating production systems. Achieving this goal should have a profound effect on the human economy. Since complete auxon systems can grow exponentially, they can reach any desired size in a short time. As a result the investment of human labor into generating a specific output is no longer proportional to the size of the output or the number of steps required in producing it. Instead, the human input is only related to the difficulty of devising a new production chain to be incorporated into the auxon system. Thus, auxon systems can provide a practically unlimited supply of energy, raw materials and basic manufactured goods. Auxon systems can also be made large enough to deal with environmental problems like air pollution or climate changes on a global scale. Indeed, the global warming caused by carbon dioxide emissions from human activities over the last centuries could be arrested with auxon technology. Removal of the excess carbon dioxide from the air could be accomplished by an auxon colony in about a decade. 
We have identified a series of high temperature processes that can separate raw dirt into its chemical components, subject to the constraints that no rare elements may be used in the processing. We believe that the processing is sufficiently robust to allow for natural variations in the chemical composition of the source material. The conceptual design of the process is outlined in Figure 1. In summary we are using first silicon and later carbon as a reducing agent to extract the elements from a mixture of minerals that reflects the average composition of the crust. We have limited the carbothermic reduction to those steps where carbon is the only possible reducing agent, i.e. $\mathrm{CaO}, \mathrm{Al}_{2} \mathrm{O}_{3}$ and $\mathrm{SiO}$. The reason for this is that in comparison to silicon, carbon is much less abundant. As we found out from the literature, the carbothermic processes are relatively well understood. Reduction with silicon, however, has not been very much explored in the past. Consequently we have focused our effort on those steps in the diagram that use silicon to affect the reaction. We include in this the reduction of $\mathrm{SiO}_{2}$ with $\mathrm{Si}$ to $\mathrm{SiO}$.

Our first step has been to obtain typical average compositions of crustal rock and to develop a set of samples that mirror these abundances. We proceeded to develop an improved understanding of the thermodynamics of the process and elucidated in some detail the role of silicates in the process. A potential stumbling block to the process as sketched out in Figure 1, is the binding of $\mathrm{SiO}_{2}$ to the other components of the melt. In the solid state this plays a major role. The free energy effect is substantial and when we started out, it was not clear how this would affect the overall process. The size of the effect in the melt has been debated in the literature. Our results have shown that the process we developed can proceed in spite of this correction in free energy. We have also found the temperatures proposed in the original scheme are higher than necessary, if one is willing to introduce relatively simple, low-grade, vacuum systems.

Specifically we studied the reduction of iron in a mineral using $\mathrm{Si}$ as the reducing agent. Silicon and synthetic dirt with typical iron content was heated in a small furnace above the melting temperature of iron. We found that the mineral powder and the silicon would react, forming an iron rich silicon melt, which once saturated would precipitate out as nearly pure iron liquid. The iron melt, due to its high surface tension would form small droplets that collected at the bottom of the crucible.

Our first experiments were performed in a conventional furnace with handmade crucibles, designed to prevent air from accessing the samples. In effect we surrounded the small sample crucibles with a reducing agent that prevented air from accessing the small reaction vessel. For the next phase of the experiments we used a vacuum system that gave us much more control over our environment. We spend a large effort on designing and developing a small vacuum furnace with a charge of less than $1 \mathrm{~cm}^{3}$. This furnace could 
reach temperatures of more than $1800^{\circ} \mathrm{C}$ and allowed us to perform experiments on mineral mixtures designed to probe the extraction of $\mathrm{SiO}$ from the process.

The result demonstrates that the original chemistry as represented in Figure 1 is feasible. For example, we have shown that the presence of aluminum oxides and calcium oxides does not prevent the volatilization of $\mathrm{SiO}_{2}$ with $\mathrm{Si}$. We have demonstrated that we can collect substantial amounts of $\mathrm{SiO}$ in our small vacuum system. The simple design of the furnace suggests that vacuum systems may provide a viable avenue for material processing. The advantage of the approach is much better control over the process and a substantial reduction in temperature as compared to the baseline process. At the higher temperature range we expect a reduction by about $300^{\circ} \mathrm{C}$. Furthermore, heat losses are easily controlled in such a system. As a result we expect that future designs will follow this approach. More work is required to carefully develop each step.

\section{Publications \& Press Response}

1. Exponential Growth of Large Self-Reproducing Machine Systems, K. S. Lackner, C. H. Wendt, Mathl. Comput. Modelling Vol, 21, NO. 10, pp. 55-81, 1995

2. Robot, build thyself, Discover Magazine, October 1995 , Special $15^{\text {th }}$ Anniversary Issue, by Thomas Bass, an eight page article describing our work.

\section{References}

[3] von Neumann, J., "Theory of Self-Reproducing Automata," (Edited and completed by A. W. Burks. University of Illinois Press, Urbana, IL, (1966).

[4] Moore E. F., “Artificial Living Plants,” Scientific American, 195 (4), 118-126, (October 1956).

[5] Dyson F., Disturbing the Universe, Chapter 18: "Thought Experiments" which is based on the 1970 Vanuxem Lecture held at Princeton, Harper \& Row, New York (1979)

[6] Long, J. E. and T. J. Healy, “Advanced automation for space mission: Technical Summary, Report of the 1980 NASA/ASEE Summer Study on the Feasibility of Using Machine Intelligence in Space Applications," University of Santa Clara, (1980).

[7] Freitas, Jr., F. A. and W. B. Zachary, "A self-replicating, growing lunar factory," in Proceedings of the Fifth Princeton/AIAA Conference, (Edited by J. Grey and L. A. Hamdan), pp. 109-119, American Institute of Aeronautics and Astronautics, New York (May 1981). 


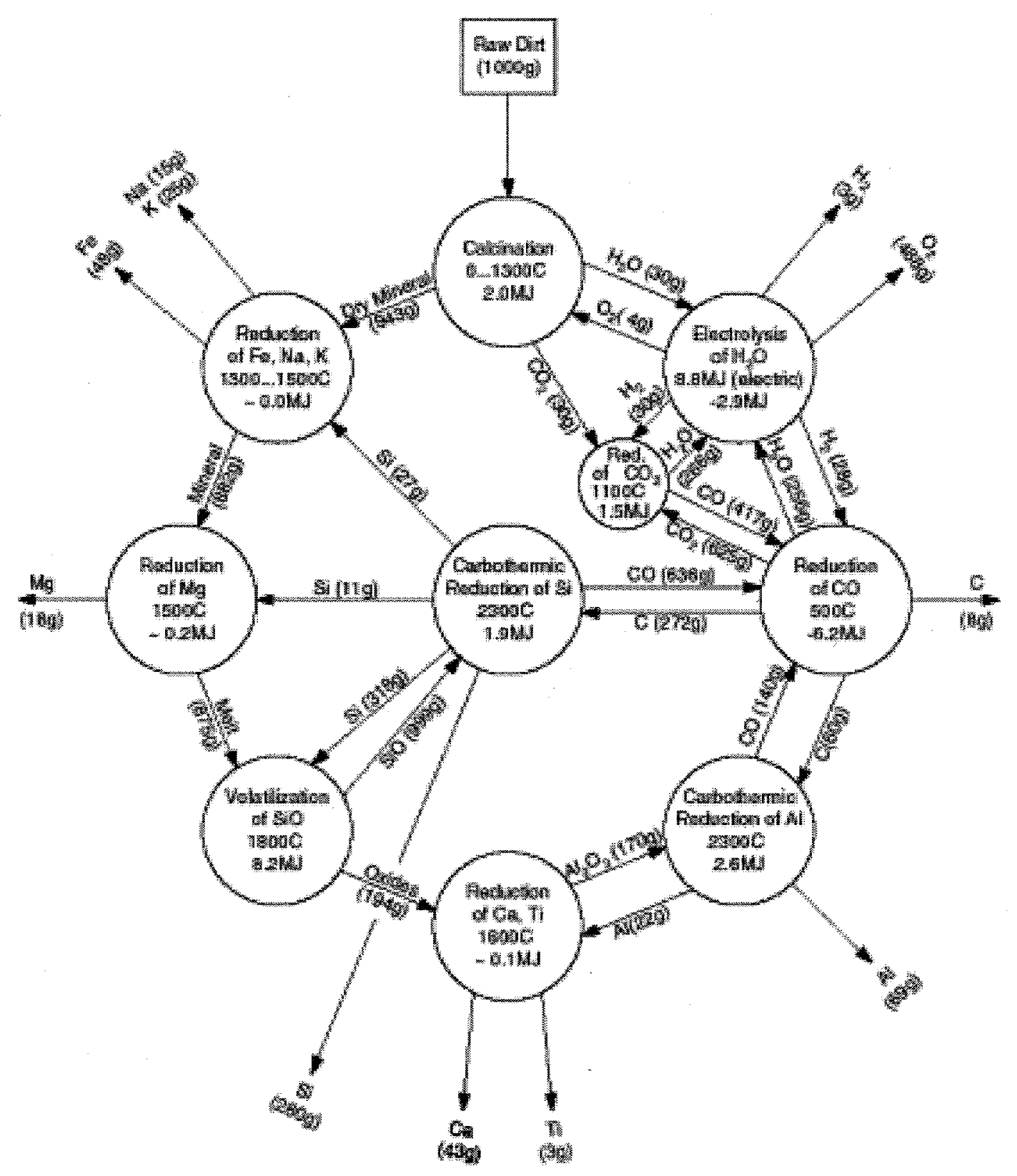

Figure 1: The element separation cycle, from Ref. 1. Processing begins with raw dirt or soil as input at the top of the figure, and proceeds counterclockwise. The products are shown at the outside. With each step is listed the amount of heat which must be added to bring the reactants up to the process temperature and to accomplish the reaction. The heats are normalized to $1 \mathrm{~kg}$ of raw dirt. The electrolysis step requires electric energy, which is listed separately. Ignoring recovery of heat from the final products and steps with a net energy release, the total energy consumption of the cycle is $25 \mathrm{MJ} / \mathrm{kg}$. The thermodynamic calculations were performed for atmospheric pressure. Note that processing in a vacuum will lower the temperature requirement of the high temperature processing steps by about 200 to $300^{\circ} \mathrm{C}$. 